\title{
Optimal feedforward torque control for nonlinear induction machines considering stator $\&$ rotor copper losses and current $\&$ voltage limits
}

\author{
Andre Thommessen, Christoph Hackl \\ Laboratory for Mechatronic and Renewable Energy Systems (LMRES) \\ Munich University of Applied Sciences (MUAS) \\ Munich, Germany \\ $\{$ andre.thommessen, christoph.hackl\}@hm.edu
}

\begin{abstract}
In order to analytically solve the optimal feedforward torque control (OFTC) problem of induction machines (IMs), the unified theory for synchronous machine introduced in [1] is extended by considering relevant IM nonlinearities and incorporating stator and rotor copper losses. Instead of the well known Maximum Torque per (stator) Current (MTPC) operation strategy, Maximum Torque per (copper) Losses $\left(\mathrm{MTPL}_{\mathrm{Cu}}\right)$ is realized and extended by the Maximum (rotor) Current $\left(\mathrm{MC}_{\mathrm{r}, \text { ext }}\right)$ strategy due to stator and rotor current limitations. Modeling magnetic saturation and cross-coupling effects leads to a constrained nonlinear optimization problem which is solved based on the idea of sequential quadratic programming (SQP). The second order Taylor approximations are formulated in implicit form as quadrics. Applying the Lagrangian formalism to the quadratic problem leads to analytical solution for the optimal rotor currents. For a doubly-fed induction machine (DFIM), a decision tree for optimal operation management is presented and the OFTC is validated in simulations for a real nonlinear IM.

Index Terms - efficiency, copper losses, (doubly-fed) induction machine, Maximum Torque per (copper) Losses $\left(\mathbf{M T P L}_{\mathrm{Cu}}\right)$, Maximum (rotor) Current $\left(\mathrm{MC}_{\mathrm{r} \text {, ext }}\right)$, analytical solution, sequential quadratic programming (SQP), quadrics, Lagrangian optimization, operation management
\end{abstract}

Notation $\mathbb{N}, \mathbb{R}:$ natural, real numbers. $\boldsymbol{x}:=$ $\left(x_{1}, \ldots, x_{n}\right)^{\top} \in \mathbb{R}^{n}$ : column vector, $n \in \mathbb{N}$ where “Т," and ":=" mean "transposed" and "is defined as", respectively. $\boldsymbol{a}^{\top} \boldsymbol{b}:=a_{1} b_{1}+\cdots+a_{n} b_{n}$ : scalar product of vectors $\boldsymbol{a}$ and $\boldsymbol{b} .\|\boldsymbol{x}\|:=\sqrt{\boldsymbol{x}^{\top} \boldsymbol{x}}=\sqrt{x_{1}^{2}+\cdots+x_{n}^{2}}$ : Euclidean norm of $\boldsymbol{x} . \boldsymbol{X} \in \mathbb{R}^{n \times m}$ : matrix with $n$ rows and $m$ columns. $\boldsymbol{I}_{n} \in \mathbb{R}^{n \times n}:=\operatorname{diag}(1, \ldots, 1)$ : identity matrix. $\boldsymbol{O}_{n \times m} \in \mathbb{R}^{n \times m}$ : zero matrix. $\mathbf{0}_{n} \in \mathbb{R}^{n}$ : zero vector. Park transformation angle $\phi_{\mathrm{p}} \in \mathbb{R}$ and $\boldsymbol{J}:=\left[\begin{array}{cc}0 & -1 \\ 1 & 0\end{array}\right]$ : counter-clock wise rotation matrix by $\frac{\pi}{2}$. "s.t.": subject to (optimization with constraints). $\mathbb{X} \cap \mathbb{Y}$ : intersection of sets $\mathbb{X}, \mathbb{Y} \subset \mathbb{R}^{2}$.

\section{INTRODUCTION}

About $50-70 \%$ of all electricity is consumed by electric machines with an overwhelming share of $90 \%$ induction machines (IMs) [2]. Moreover, IMs are also used for converterbased power generation which is the focus of this paper: Doubly-fed induction machines (DFIMs) with grid-connected stator and inverter-fed rotor as widely used in wind energy conversion systems (WECS) [3]. However, the proposed generic approach can also be applied to other types of inverterfed IMs, such as squirrel-cage induction machines (SCIMs).

The highest priority of the OFTC is to provide the reference torque while minimizing losses. In a wide operating range, there usually exist (infinitely many) different combinations of reference currents resulting in the same torque. Thus, an optimal reference current computation (ORCC) is desirable which minimizes the current-dependent IM losses while reaching the reference torque and taking into account operating (e.g. current $\&$ voltage) limits. To do so, this paper proposes a physicsbased nonlinear IM model for analytical ORCC.

In [4-7] the efficiency of DFIM-based WECS is increased based on an improved reactive power sharing between the rotor and the stator circuit considering the dominant copper losses (also iron losses in [4], [6] and converter losses in [6]). Neglecting the impact of the active rotor current, the reactive rotor current calculation in [6] results in a power saving of $15 \%$ of the total losses for low load operation. In [8], a new structure for WECS is proposed which also allows to regulate the DFIM stator voltage without an additional converter for further efficiency improvement based on flux weakening. However, all analytical reference current computations stated above neglect nonlinear saturation and cross-coupling effects and operating limits.

In [9], saturation effects are modeled by a current dependent but scalar magnetizing inductance. The numerically and offline calculated reference currents are saved in lookup-tables (LUTs) for OFTC. In [10], an IM identification method based on measurements is used to extract optimal reference current LUTs. As this method requires expensive torque sensors, [10] proposes the MTPC strategy as alternative. However, MTPC only minimizes the copper losses of the primary (inverterfed) side and not of the secondary (short-circuited or gridconnected) side. Moreover, the step-wise or numerical calculations of the LUT-based approaches result in limited accuracy. Since the copper and iron losses depend on temperatures at different locations, [9] proposes to use multiple LUTs accepting an significantly increased computational effort due to increased dimensions of the LUTs.

To the best knowledge of the authors, all existing approaches 
to solve the OFTC problem for IMs are either based on simplified IM models or on LUT-based approaches using numerical calculations or expensive measurements. Thus, this paper provides an advanced nonlinear IM model for OFTC which allows for analytical ORCC taking into account (i) nonlinear magnetic saturation, (ii) cross-coupling effects (i.e. the dependencies between orthogonal current and magnetic flux vectors are not neglected), and (iii) stator and rotor copper losses. In addition, the proposed OFTC approach is generic as it allows further (online) parameter adaption, such as the temperature dependent stator and rotor resistances or even iron losses [11]. The nonlinear IM model is based on magnetic flux linkage maps obtained by measurements [10] or finite element analysis (FEA) [12]. The focus of this paper is on minimizing the total IM copper losses assuming that the iron losses are negligible or constant for a given torque as in [5].

Contributions of this paper are: The unified theory for synchronous machines presented in [1]\&[11] is extended and applied to IMs: Section II introduces the nonlinear IM model and problem statement; Section III-A integrates the measured magnetic flux linkage maps into the quadratic IM model approximation; Section III-B discusses quadratic programming with analytical solution; Section III-C proposes a novel operation management for OFTC in the full operating range (i. e. even at the operating limits); Section IV discusses implementation and simulation results.

\section{MODELING AND PROBLEM STATEMENT}

\section{A. Nonlinear IM model}

For stator angular velocity $\omega_{\mathrm{s}}:=-\omega_{\mathrm{p}}$ and rotor relative angular velocity $\omega_{\mathrm{r}}:=n_{\mathrm{p}} \omega_{\mathrm{m}}-\omega_{\mathrm{p}}$ with machine angular velocity $\omega_{\mathrm{m}}$ and number of pole pairs $n_{\mathrm{p}}$, the stator and rotor voltages in the $d q$-reference frame are given by

$$
\begin{aligned}
\underbrace{\left(\begin{array}{c}
\boldsymbol{u}_{\mathrm{s}}^{d q} \\
\boldsymbol{u}_{\mathrm{r}}^{d q}
\end{array}\right)}_{=: \boldsymbol{u}^{d q}}= & \underbrace{\left[\begin{array}{cc}
\boldsymbol{R}_{\mathrm{s}}^{d q} & \boldsymbol{O}_{2 \times 2} \\
\boldsymbol{O}_{2 \times 2} & \boldsymbol{R}_{\mathrm{r}}^{d q}
\end{array}\right]}_{=: \boldsymbol{R}^{d q}} \underbrace{\left(\begin{array}{c}
\boldsymbol{i}_{\mathrm{s}}^{d q} \\
\boldsymbol{i}_{\mathrm{r}}^{d q}
\end{array}\right)}_{=: \boldsymbol{i}^{d q}}+\frac{\mathrm{d}}{\mathrm{d} t} \underbrace{\left(\begin{array}{c}
\boldsymbol{\psi}_{\mathrm{s}}^{d q} \\
\boldsymbol{\psi}_{\mathrm{r}}^{d q}
\end{array}\right)}_{=: \boldsymbol{\psi}^{d q}} \\
& -\underbrace{\left[\begin{array}{cc}
\omega_{\mathrm{s}} & 0 \\
0 & \omega_{\mathrm{r}}
\end{array}\right]}_{=: \boldsymbol{\Omega}} \underbrace{\left[\begin{array}{cc}
\boldsymbol{J} & \boldsymbol{O}_{2 \times 2} \\
\boldsymbol{O}_{2 \times 2} & \boldsymbol{J}
\end{array}\right]}_{=: \boldsymbol{J}_{2}} \underbrace{\left(\begin{array}{c}
\boldsymbol{\psi}_{\mathrm{s}}^{d q} \\
\boldsymbol{\psi}_{\mathrm{r}}^{d q}
\end{array}\right)}_{=: \boldsymbol{\psi}^{d q}} \\
= & \boldsymbol{R}^{d q} \boldsymbol{i}^{d q}-\boldsymbol{\Omega} \boldsymbol{J}_{2} \boldsymbol{\psi}^{d q}+\frac{\mathrm{d}}{\mathrm{d} t} \boldsymbol{\psi}^{d q}
\end{aligned}
$$

with voltages $\boldsymbol{u}_{\mathrm{s}}^{d q}:=\left(u_{\mathrm{s}}^{d}, u_{\mathrm{s}}^{q}\right)^{\top}, \boldsymbol{u}_{\mathrm{r}}^{d q}:=\left(u_{\mathrm{r}}^{d}, u_{\mathrm{r}}^{q}\right)^{\top}$, flux linkages $\psi_{\mathrm{s}}^{d q}:=\left(\psi_{\mathrm{s}}^{d}, \psi_{\mathrm{s}}^{q}\right)^{\top}, \psi_{\mathrm{r}}^{d q}:=\left(\psi_{\mathrm{r}}^{d}, \psi_{\mathrm{r}}^{q}\right)^{\top}$, currents $\boldsymbol{i}_{\mathrm{s}}^{d q}:=\left(i_{\mathrm{s}}^{d^{d}}, i_{\mathrm{s}}^{q}\right)^{\top}, \boldsymbol{i}_{\mathrm{r}}^{d q}:=\left(i_{\mathrm{r}}^{d}, i_{\mathrm{r}}^{q}\right)^{\top}$ of stator and rotor, respectively. Moreover, $\boldsymbol{R}_{\mathrm{s}}^{d q}, \boldsymbol{R}_{\mathrm{r}}^{d q}$ denote the resistance matrix (both $\in \mathbb{R}^{2 \times 2}$ ) of stator and rotor, respectively, which simplify to $\boldsymbol{R}_{\mathrm{s}}^{d q}=R_{\mathrm{s}} \boldsymbol{I}_{2}, \boldsymbol{R}_{\mathrm{r}}^{d q}=R_{\mathrm{r}} \boldsymbol{I}_{2}$ for symmetric, fault-free windings. Using equal sign conventions for $\omega_{\mathrm{m}}$ and the torque, latter is given by

$$
m_{\mathrm{m}}=-\underbrace{\frac{2}{3 \kappa^{2}}}_{=: \kappa_{\mathrm{p}}} n_{\mathrm{p}}\left(\boldsymbol{i}_{\mathrm{r}}^{d q}\right)^{\top} \boldsymbol{J} \boldsymbol{\psi}_{\mathrm{r}}^{d q}=-\kappa_{\mathrm{p}} n_{\mathrm{p}}\left(\boldsymbol{i}_{\mathrm{r}}^{d q}\right)^{\top} \boldsymbol{J} \boldsymbol{\psi}_{\mathrm{r}}^{d q}
$$

with Clarke transform scaling factor $\kappa$ (e.g. $\kappa:=\frac{2}{3}$ for amplitude-invariant transformation).

Neglecting iron losses, the IM losses $p_{\mathrm{m}, \mathrm{L}}$ are copper losses $p_{\mathrm{Cu}}$ comprising stator and rotor copper losses, i. e.

$$
p_{\mathrm{m}, \mathrm{L}}:=p_{\mathrm{Cu}}:=\underbrace{\kappa_{\mathrm{p}}\left(\boldsymbol{i}_{\mathrm{s}}^{d q}\right)^{\top} \boldsymbol{R}_{\mathrm{s}}^{d q} \boldsymbol{i}_{\mathrm{s}}^{d q}}_{=: p_{\mathrm{s}, \mathrm{Cu}}}+\underbrace{\kappa_{\mathrm{p}}\left(\boldsymbol{i}_{\mathrm{r}}^{d q}\right)^{\top} \boldsymbol{R}_{\mathrm{r}}^{d q} \boldsymbol{i}_{\mathrm{r}}^{d q}}_{=: p_{\mathrm{r}, \mathrm{Cu}}} .
$$

\section{B. Problem statement}

In the following, the typical DFIM configuration of WECS is considered but the present approach is generic and can also be applied to other IM types (e. g. SCIMs by setting the secondary (short-circuited) terminal voltage to zero).

The goal of OFTC is to determine the rotor current reference $i_{\mathrm{r}, \mathrm{ref}}^{d q}$ which reaches the reference torque $m_{\mathrm{m} \text {,ref }}$ with minimal IM losses taking into account current and voltage limits. Thus, the optimization problem can be stated as

$$
\left.\begin{array}{rl}
\boldsymbol{i}_{\mathrm{r}, \mathrm{ref}}^{d q} & :=\underset{\boldsymbol{i}_{\mathrm{r}}^{d q} \in \mathbb{S}}{\arg \min } p_{\mathrm{m}, \mathrm{L}}\left(\boldsymbol{i}_{\mathrm{r}}^{d q}\right) \\
m_{\mathrm{m}} & =\bar{m}_{\mathrm{m}, \mathrm{ref}}:=\operatorname{sat}_{m_{\mathrm{m}, \text { min }}}^{m_{\mathrm{max}}}\left(m_{\mathrm{m}, \mathrm{ref}}\right)
\end{array}\right\}
$$

with the feasible set

$$
\begin{aligned}
\mathbb{S}:=\left\{\boldsymbol{i}_{\mathrm{r}, \mathrm{ref}}^{d q} \in \mathbb{R}^{2} \mid \frac{2}{3 \kappa}\left\|\boldsymbol{i}_{\mathrm{s}}^{d q}\right\|\right. & \leq \widehat{\imath}_{\mathrm{s}, \max } \wedge \frac{2}{3 \kappa}\left\|\boldsymbol{i}_{\mathrm{r}}^{d q}\right\| \leq \widehat{\imath}_{\mathrm{r}, \max } \wedge \\
\frac{2}{3 \kappa}\left\|\boldsymbol{u}_{\mathrm{s}}^{d q}\right\| & \left.\leq \widehat{u}_{\mathrm{s}, \max } \wedge \frac{2}{3 \kappa}\left\|\boldsymbol{u}_{\mathrm{r}}^{d q}\right\| \leq \widehat{u}_{\mathrm{r}, \max }\right\} .
\end{aligned}
$$

The corresponding maximal admissible values are indicated by the subscript 'max'.

\section{PROPOSED SOLUTION}

To solve the constrained nonlinear optimization problem (4), the general idea of sequential quadratic programming (SQP) [13] and, in particular, the sequence used in [1] \& [11] is adopted which consists of three main stages per sequence: (i) quadratic approximation of the loss function and all constraints at the current operating point, (ii) applying the Lagrangian formalism to solve the quadratic (sub-)problems (QPs), (iii) selection of the optimal solution and the operating point for the next iteration. The sequence terminates if the solutions of the QP converged to an operating point which is equal to the local or global minimizer for a convex differentiable objective function [13].

In order to obtain the quadratic approximations, analytical differentiable model functions are required. However, due to magnetic saturation, the magnetic flux linkages are nonlinear and usually unknown functions of the machine currents, i.e. $\boldsymbol{\psi}^{d q}=\boldsymbol{\psi}^{d q}\left(\boldsymbol{i}_{\mathrm{s}}^{d q}, \boldsymbol{i}_{\mathrm{r}}^{d q}\right)$. Thus, the presented IM model is based on magnetic flux linkage maps. Considering the steady-state magnetic flux linkages as functions of the rotor current only, i.e.

$$
\underbrace{\left(\begin{array}{c}
\widetilde{\boldsymbol{\psi}}_{\mathrm{r}}^{d q}\left(\boldsymbol{i}_{\mathrm{r}}^{d q}\right) \\
\widetilde{\boldsymbol{\psi}}_{\mathrm{s}}^{d q}\left(\boldsymbol{i}_{\mathrm{r}}^{d q}\right)
\end{array}\right)}_{=: \widetilde{\boldsymbol{\psi}}^{d q}\left(\boldsymbol{i}_{\mathrm{r}}^{d q}\right)}:=\underbrace{\left(\begin{array}{c}
\boldsymbol{\psi}_{\mathrm{s}}^{d q}\left(\boldsymbol{i}_{\mathrm{s}}^{d q}\left(\boldsymbol{i}_{\mathrm{r}}^{d q}\right), \boldsymbol{i}_{\mathrm{r}}^{d q}\right) \\
\boldsymbol{\psi}_{\mathrm{r}}^{d q}\left(\boldsymbol{i}_{\mathrm{s}}^{d q}\left(\boldsymbol{i}_{\mathrm{r}}^{d q}\right), \boldsymbol{i}_{\mathrm{r}}^{d q}\right)
\end{array}\right)}_{=: \boldsymbol{\psi}^{d q}\left(\boldsymbol{i}_{\mathrm{s}}^{d q}\left(\boldsymbol{i}_{\mathrm{r}}^{d q}\right), \boldsymbol{i}_{\mathrm{r}}^{d q}\right)},
$$


the linearization of the flux linkages can be stated as

$$
\boldsymbol{\psi}^{d q}=\widetilde{\boldsymbol{\psi}}^{d q}\left(\boldsymbol{i}_{\mathrm{r}}^{d q}\right) \approx \underline{\widetilde{\mathcal{L}}}^{d q} \underbrace{\left(\boldsymbol{i}_{\mathrm{r}}^{d q}-\overline{\boldsymbol{i}}_{\mathrm{r}}^{d q}\right)}_{=: \Delta \boldsymbol{i}_{\mathrm{r}}^{d q}}+\underbrace{\widetilde{\boldsymbol{\psi}}^{d q}\left(\overline{\boldsymbol{i}}_{\mathrm{r}}^{d q}\right)}_{=: \overline{\boldsymbol{\psi}}^{d q}}
$$

where

$$
\underline{\widetilde{\mathcal{L}}}^{d q}:=\left[\begin{array}{c}
\widetilde{\mathcal{M}}_{\mathrm{s}, \mathrm{r}}^{d q} \\
\widetilde{\widetilde{\mathcal{L}}}_{\mathrm{r}}^{d q}
\end{array}\right]:=\left.\left[\begin{array}{l}
\frac{\partial \widetilde{\boldsymbol{\psi}}_{\mathrm{s}}^{d q}\left(\boldsymbol{i}_{\mathrm{r}}^{d q}\right)}{\left.\partial \boldsymbol{i}_{\mathrm{r}}^{d q}\right)} \\
\frac{\partial \widetilde{\boldsymbol{\psi}}_{\mathrm{r}}^{d q}\left(\boldsymbol{i}_{\mathrm{r}}{ }^{d q}\right)}{\partial\left(\boldsymbol{i}_{\mathrm{r}}^{d q}\right)^{\top}}
\end{array}\right]\right|_{\boldsymbol{i}_{\mathrm{r}}^{d q}=\overline{\boldsymbol{i}}_{\mathrm{r}}^{d q}} \in \mathbb{R}^{4 \times 2} .
$$

Note that all quantities evaluated at the current operating point are denoted by an overline or underline for symbols with tilde. The maps of the rotor-side magnetic flux linkages \& steadystate differential inductances are shown in Fig. $1 \& 2$ whereas the stator-side maps are not shown due to space restrictions.

\section{A. Definition of machine quadrics}

Applying the second-order Taylor series expansion ${ }^{1}$

$f(\boldsymbol{x}) \approx f(\overline{\boldsymbol{x}})+\left(\left.\frac{\mathrm{d} f(\boldsymbol{x})}{\mathrm{d} \boldsymbol{x}}\right|_{\overline{\boldsymbol{x}}}\right)^{\top}(\boldsymbol{x}-\overline{\boldsymbol{x}})+\frac{1}{2}(\boldsymbol{x}-\overline{\boldsymbol{x}})^{\top}\left[\left.\frac{\mathrm{d}^{2} f(\boldsymbol{x})}{\mathrm{d} \boldsymbol{x} \mathrm{d} \boldsymbol{x}^{\top}}\right|_{\overline{\boldsymbol{x}}}\right](\boldsymbol{x}-\overline{\boldsymbol{x}})$,

to all relevant functions of the optimization problem (4) leads to implicit formulations as (approximated) quadrics. The corresponding set of an arbitrary quadric with $\boldsymbol{A} \in \mathbb{R}^{2 \times 2}$, $a \in \mathbb{R}^{2 \times 1}, \alpha \in \mathbb{R}$ and $\boldsymbol{x} \in \mathbb{R}^{2 \times 1}$ is given by

$$
\mathbb{Q}_{\mathrm{A}}:=\left\{\boldsymbol{x} \in \mathbb{R}^{2} \mid(\boldsymbol{x})^{\top} \boldsymbol{A} \boldsymbol{x}+2 \boldsymbol{a}^{\top} \boldsymbol{x}+\alpha=0\right\} .
$$

In steady state, i.e.

$$
\begin{aligned}
\frac{\mathrm{d}}{\mathrm{d} t} \boldsymbol{\psi}^{d q}=\mathbf{0}_{4} & \stackrel{(1)}{\Rightarrow} \boldsymbol{u}^{d q}=\boldsymbol{R}^{d q} \boldsymbol{i}^{d q}-\boldsymbol{\Omega} \boldsymbol{J}_{2} \boldsymbol{\psi}^{d q} \\
& \Rightarrow \boldsymbol{i}_{\mathrm{s}}^{d q}=\left(\boldsymbol{R}_{\mathrm{s}}^{d q}\right)^{-1}\left(\boldsymbol{u}_{\mathrm{s}}^{d q}+\omega_{\mathrm{s}} \boldsymbol{J} \boldsymbol{\psi}_{\mathrm{s}}^{d q}\right),
\end{aligned}
$$

the (approximated) stator copper loss quadric is given by

$$
\begin{aligned}
& \frac{p_{\mathrm{s}, \mathrm{Cu}}}{\kappa_{\mathrm{p}}}=\left(\boldsymbol{i}_{\mathrm{s}}^{d q}\right)^{\top} \boldsymbol{R}_{\mathrm{s}}^{d q} \boldsymbol{i}_{\mathrm{s}}^{d q}
\end{aligned}
$$

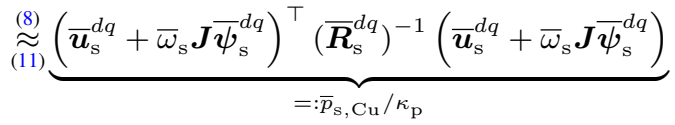

$$
\begin{aligned}
& +2 \underbrace{\left(\overline{\boldsymbol{u}}_{\mathrm{s}}^{d q}+\bar{\omega}_{\mathrm{s}} \boldsymbol{J} \overline{\boldsymbol{\psi}}_{\mathrm{s}}^{d q}\right)^{\top}\left(\overline{\boldsymbol{R}}_{\mathrm{s}}^{d q}\right)^{-1} \bar{\omega}_{\mathrm{s}} \boldsymbol{J} \underline{\widetilde{\mathcal{M}}}_{\mathrm{s}, \mathrm{r}}^{d q}}_{=: i_{\mathrm{s}, \mathrm{Cu}}^{*} / \kappa_{\mathrm{p}}} \Delta \boldsymbol{i}_{\mathrm{r}}^{d q}
\end{aligned}
$$

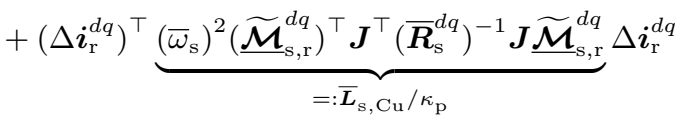

$$
\begin{aligned}
& \Rightarrow 0 \approx\left(\boldsymbol{i}_{\mathrm{r}}^{d q}\right)^{\top} \overline{\boldsymbol{L}}_{\mathrm{s}, \mathrm{Cu}} \boldsymbol{i}_{\mathrm{r}}^{d q}+2 \underbrace{\left(\overline{\boldsymbol{l}}_{\mathrm{s}, \mathrm{Cu}}^{* \top}-\left(\overline{\boldsymbol{i}}_{\mathrm{r}}^{d q}\right)^{\top} \overline{\boldsymbol{L}}_{\mathrm{s}, \mathrm{Cu}}\right)}_{=: \overline{\boldsymbol{l}}_{\mathrm{s}, \mathrm{Cu}}^{\top}} \boldsymbol{i}_{\mathrm{r}}^{d q} \\
& +\underbrace{\bar{p}_{\mathrm{s}, \mathrm{Cu}}-2 \overline{\boldsymbol{l}}_{\mathrm{s}, \mathrm{Cu}}^{* \top} \overline{\boldsymbol{i}}_{\mathrm{r}}^{d q}+\left(\overline{\boldsymbol{i}}_{\mathrm{r}}^{d q}\right)^{\top} \overline{\boldsymbol{L}}_{\mathrm{s}, \mathrm{Cu}} \overline{\boldsymbol{i}}_{\mathrm{r}}^{d q}-p_{\mathrm{s}, \mathrm{Cu}}}_{=: \rho_{\mathrm{s}, \mathrm{Cu}}} .
\end{aligned}
$$

${ }^{1}$ For some $\boldsymbol{M} \in \mathbb{R}^{n \times m}, \boldsymbol{g}(\boldsymbol{x}) \in \mathbb{R}^{n}$ and $\boldsymbol{h}(\boldsymbol{x}) \in \mathbb{R}^{m}$, the second-order approximation of the scalar function $f(\boldsymbol{x}):=\boldsymbol{g}(\boldsymbol{x})^{\top} \boldsymbol{M} \boldsymbol{h}(\boldsymbol{x})$ is given by (8) with gradient $\left(\frac{\mathrm{d} f(\boldsymbol{x})}{\mathrm{d} \boldsymbol{x}}\right)^{\top}=\boldsymbol{h}(\boldsymbol{x})^{\top} \boldsymbol{M}^{\top}\left[\frac{\mathrm{d} \boldsymbol{g}(\boldsymbol{x})}{\mathrm{d} \boldsymbol{x}^{\top}}\right]+\boldsymbol{g}(\boldsymbol{x})^{\top} \boldsymbol{M}\left[\frac{\mathrm{d} \boldsymbol{h}(\boldsymbol{x})}{\mathrm{d} \boldsymbol{x}}\right]$ and symmetric Hessian matrix $\left[\frac{\mathrm{d}^{2} f(\boldsymbol{x})}{\mathrm{d} \boldsymbol{x} \mathrm{d} \boldsymbol{x}}\right] \approx\left[\frac{\mathrm{d} \boldsymbol{h}(\boldsymbol{x})^{\top}}{\mathrm{d} \boldsymbol{x}}\right] \boldsymbol{M}^{\top}\left[\frac{\mathrm{d} \boldsymbol{g}(\boldsymbol{x})}{\mathrm{d} \boldsymbol{x}}\right]+$ $\left[\frac{\mathrm{d} \boldsymbol{g}(\boldsymbol{x})^{\top}}{\mathrm{d} \boldsymbol{x}}\right] \boldsymbol{M}\left[\frac{\mathrm{d} \boldsymbol{h}(\boldsymbol{x})}{\mathrm{d} \boldsymbol{x}^{\top}}\right]$ where $\frac{\mathrm{d}}{\mathrm{d} \boldsymbol{x}}\left[\frac{\mathrm{d} \boldsymbol{g}(\boldsymbol{x})}{\mathrm{d} \boldsymbol{x}^{\top}}\right]$ and $\frac{\mathrm{d}}{\mathrm{d} \boldsymbol{x}}\left[\frac{\mathrm{d} \boldsymbol{h}(\boldsymbol{x})}{\mathrm{d} \boldsymbol{x}^{\top}}\right]$ are neglected (for details see [14]).

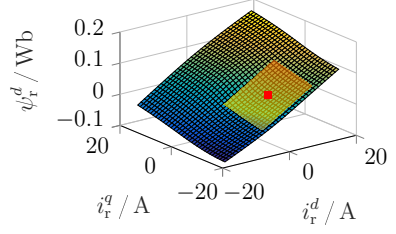

(a) Rotor $d$-flux linkage.

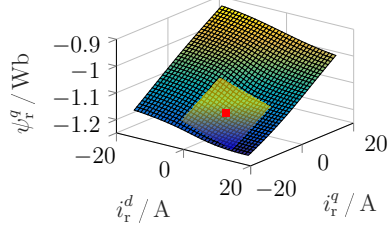

(b) Rotor $q$-flux linkage.
Figure 1: Nonlinear magnetic flux linkages $\boldsymbol{\psi}_{\mathrm{r}}^{d q}$ in the stator voltage aligned $d q$-reference frame, i.e. $u_{\mathrm{s}}^{q}=0$, of a real DFIM with tangent plane $[\square]$ at some operating point $\overline{\boldsymbol{i}}_{\mathrm{r}}^{d q}[\boldsymbol{\square}]$.

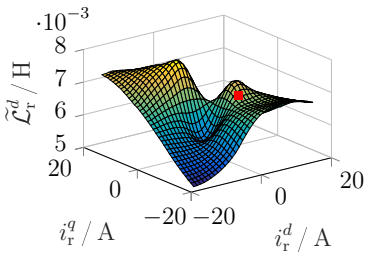

(a) $\widetilde{\mathcal{L}}_{\mathrm{r}}^{d}:=\frac{\partial \widetilde{\psi}_{\mathrm{r}}^{d}\left(i_{\mathrm{r}}^{d q}\right)}{\partial i_{\mathrm{r}}^{d}}$

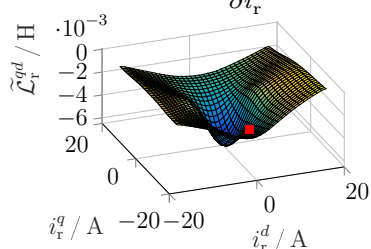

(c) $\widetilde{\mathcal{L}}_{\mathrm{r}}^{q d}:=\frac{\partial \widetilde{\psi}_{\mathrm{r}}^{q}\left(i_{\mathrm{r}}^{d q}\right)}{\partial i_{\mathrm{r}}^{d}}$

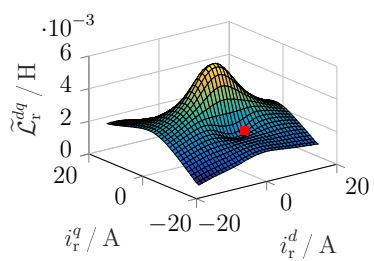

(b) $\widetilde{\mathcal{L}}_{\mathrm{r}}^{d q}:=\frac{\partial \widetilde{\psi}_{\mathrm{r}}^{d}\left(i_{\mathrm{r}}^{d q}\right)}{\partial i_{\mathrm{r}}^{q}}$.

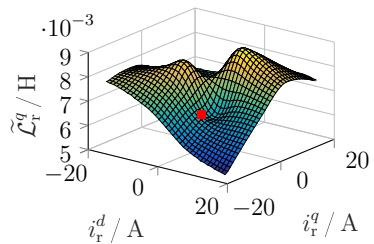

(d) $\widetilde{\mathcal{L}}_{\mathrm{r}}^{q}:=\frac{\partial \widetilde{\psi}_{\mathrm{r}}^{q}\left(i_{\mathrm{r}}^{d q}\right)}{\partial i_{\mathrm{r}}^{q}}$
Figure 2: Nonlinear steady-state differential inductances $\widetilde{\mathcal{L}}_{\mathrm{r}}^{d q}\left(\boldsymbol{i}_{\mathrm{r}}^{d q}\right)$ of a real DFIM with some operating point $\overline{\boldsymbol{i}}_{\mathrm{r}}^{d q}\left[{ }^{[}\right]$.

Adding to (12) the rotor copper loss quadric

$$
0=\left(\boldsymbol{i}_{\mathrm{r}}^{d q}\right)^{\top} \underbrace{\kappa_{\mathrm{p}} \boldsymbol{R}_{\mathrm{r}}^{d q}}_{=: \overline{\boldsymbol{L}}_{\mathrm{r}, \mathrm{Cu}}} \boldsymbol{i}_{\mathrm{r}}^{d q} \underbrace{-p_{\mathrm{r}, \mathrm{Cu}}}_{=: \rho_{\mathrm{r}, \mathrm{Cu}}},
$$

the quadric of the total loss objective function (3) is given by

$$
\begin{gathered}
\stackrel{(3)}{\Rightarrow} \underset{(13)}{\underset{(12)}{\approx}}\left(\boldsymbol{i}_{\mathrm{r}}^{d q}\right)^{\top} \underbrace{\left[\overline{\boldsymbol{L}}_{\mathrm{r}, \mathrm{Cu}}+\overline{\boldsymbol{L}}_{\mathrm{s}, \mathrm{Cu}}\right]}_{=: \overline{\boldsymbol{L}}_{\mathrm{Cu}}} \boldsymbol{i}_{\mathrm{r}}^{d q}+2 \underbrace{\overline{\boldsymbol{l}}_{\mathrm{s}, \mathrm{Cu}}^{\top}}_{=: \overline{\boldsymbol{l}}_{\mathrm{Cu}}^{\top}} \boldsymbol{i}_{\mathrm{r}}^{d q} \\
+\underbrace{\rho_{\mathrm{s}, \mathrm{Cu}}+\rho_{\mathrm{r}, \mathrm{Cu}}}_{=: \rho_{\mathrm{Cu}}} .
\end{gathered}
$$

The equality constraint in (4) leads to the torque quadric

$$
\begin{aligned}
& \bar{m}_{\mathrm{m}, \mathrm{ref}} \underset{(8)}{\stackrel{(2)}{\approx}} \underbrace{-\kappa_{\mathrm{p}} n_{\mathrm{p}}\left(\overline{\boldsymbol{i}}_{\mathrm{r}}^{d q}\right)^{\top} \boldsymbol{J} \overline{\boldsymbol{\psi}}_{\mathrm{r}}^{d q}}_{=: \bar{m}_{\mathrm{m}}} \\
& +2 \underbrace{\frac{-\kappa_{\mathrm{p}} n_{\mathrm{p}}}{2}\left\{\left(\overline{\boldsymbol{\psi}}_{\mathrm{r}}^{d q}\right)^{\top} \boldsymbol{J}^{\top}+\left(\overline{\boldsymbol{i}}_{\mathrm{r}}^{d q}\right)^{\top} \boldsymbol{J} \underline{\mathcal{L}}_{\mathrm{r}}^{d q}\right\}}_{=: \overline{\boldsymbol{t}}^{* \top}} \Delta \boldsymbol{i}_{\mathrm{r}}^{d q} \\
& +\left(\Delta \boldsymbol{i}_{\mathrm{r}}^{d q}\right)^{\top} \underbrace{\frac{-\kappa_{\mathrm{p}} n_{\mathrm{p}}}{2}\left[\left(\tilde{\widetilde{\mathcal{L}}}_{\mathrm{r}}^{d q}\right)^{\top} \boldsymbol{J}^{\top}+\boldsymbol{J} \underline{\widetilde{\mathcal{L}}}_{\mathrm{r}}^{d q}\right]}_{=: \overline{\boldsymbol{T}}} \Delta \boldsymbol{i}_{\mathrm{r}}^{d q} \\
& \Rightarrow 0 \approx\left(\boldsymbol{i}_{\mathrm{r}}^{d q}\right)^{\top} \overline{\boldsymbol{T}} \boldsymbol{i}_{\mathrm{r}}^{d q}+2 \underbrace{\left(\overline{\boldsymbol{t}}^{* \top}-\left(\overline{\boldsymbol{i}}_{\mathrm{r}}^{d q}\right)^{\top} \overline{\boldsymbol{T}}\right)}_{=: \overline{\boldsymbol{t}}^{\top}} \boldsymbol{i}_{\mathrm{r}}^{d q} \\
& +\underbrace{\bar{m}_{\mathrm{m}}-2 \overline{\boldsymbol{t}}^{* \top} \overline{\boldsymbol{i}}_{\mathrm{r}}^{d q}+\left(\overline{\boldsymbol{i}}_{\mathrm{r}}^{d q}\right)^{\top} \overline{\boldsymbol{T}}_{\mathrm{r}}^{d q}-\bar{m}_{\mathrm{m}, \mathrm{ref}}}_{=: \bar{\tau}} .
\end{aligned}
$$

The quadrics of the inequality constraints in (4) are given for 
the stator current limit by

$$
\begin{aligned}
& \stackrel{(4)}{\Rightarrow} \frac{9 \kappa_{\mathrm{c}}^{2}}{4}\left(\widehat{\imath}_{\mathrm{s}, \max }\right)^{2} \geq\left(\boldsymbol{i}_{\mathrm{s}}^{d q}\right)^{\top} \boldsymbol{i}_{\mathrm{s}}^{d q}
\end{aligned}
$$

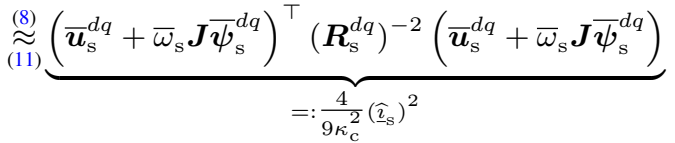

$$
\begin{aligned}
& +2 \underbrace{\left(\overline{\boldsymbol{u}}_{\mathrm{s}}^{d q}+\bar{\omega}_{\mathrm{s}} \boldsymbol{J} \overline{\boldsymbol{\psi}}_{\mathrm{s}}^{d q}\right)^{\top}\left(\boldsymbol{R}_{\mathrm{s}}^{d q}\right)^{-2} \bar{\omega}_{\mathrm{s}} \boldsymbol{J} \widetilde{\mathcal{M}}_{\mathrm{s}, \mathrm{r}}^{d q}}_{=: \frac{4}{9 \kappa_{\mathrm{c}}^{2}} \overline{\boldsymbol{c}}_{\mathrm{s}}^{* \top}} \Delta \boldsymbol{i}_{\mathrm{r}}^{d q} \\
& +\left(\Delta \boldsymbol{i}_{\mathrm{r}}^{d q}\right)^{\top} \underbrace{\left(\bar{\omega}_{\mathrm{s}}\right)^{2}\left(\underline{\boldsymbol{\mathcal { M }}}_{\mathrm{s}, \mathrm{r}}^{d q}\right)^{\top} \boldsymbol{J}^{\top}\left(\boldsymbol{R}_{\mathrm{s}}^{d q}\right)^{-2} \boldsymbol{J} \widetilde{\boldsymbol{\mathcal { M }}}_{\mathrm{s}, \mathrm{r}}^{d q}}_{=: \frac{4}{9 \kappa_{\mathrm{c}}^{2}} \overline{\boldsymbol{s}}_{\mathrm{s}}} \Delta \boldsymbol{i}_{\mathrm{r}}^{d q} \\
& \Rightarrow 0 \geq\left(\boldsymbol{i}_{\mathrm{r}}^{d q}\right)^{\top} \overline{\boldsymbol{C}}_{\mathrm{s}} \boldsymbol{i}_{\mathrm{r}}^{d q}+2 \underbrace{\left(\overline{\boldsymbol{c}}_{\mathrm{s}}^{* \top}-\left(\overline{\boldsymbol{i}}_{\mathrm{r}}^{d q}\right)^{\top} \overline{\boldsymbol{C}_{\mathrm{s}}}\right)}_{=: \overline{\boldsymbol{c}}_{\mathrm{s}}^{\top}} \boldsymbol{i}_{\mathrm{r}}^{d q} \\
& +\underbrace{\left(\widehat{\widehat{\widehat{v}}}_{\mathrm{s}}\right)^{2}-2 \overline{\boldsymbol{c}}_{\mathrm{s}}^{* \top} \overline{\boldsymbol{i}}_{\mathrm{r}}^{d q}+\left(\overline{\boldsymbol{i}}_{\mathrm{r}}^{d q}\right)^{\top} \overline{\boldsymbol{C}}_{\boldsymbol{\mathrm { s }}} \boldsymbol{\boldsymbol { i }}_{\mathrm{r}}^{d q}-\left(\widehat{\imath}_{\mathrm{s}, \max }\right)^{2}}_{=: \bar{\tau}_{\mathrm{s}}}
\end{aligned}
$$

with $\left(\boldsymbol{R}_{\mathrm{s}}^{d q}\right)^{-2}:=\left(\boldsymbol{R}_{\mathrm{s}}^{d q}\right)^{-\top}\left(\boldsymbol{R}_{\mathrm{s}}^{d q}\right)^{-1}=\left(\boldsymbol{R}_{\mathrm{s}}^{d q}\right)^{-1}\left(\boldsymbol{R}_{\mathrm{s}}^{d q}\right)^{-1}$; for the rotor current limit by

$$
\stackrel{(4)}{\Rightarrow} 0 \geq\left(\boldsymbol{i}_{\mathrm{r}}^{d q}\right)^{\top} \underbrace{\frac{4}{9 \kappa_{\mathrm{c}}^{2}} \boldsymbol{I}_{2}}_{=: C_{\mathrm{r}}} i_{\mathrm{r}}^{d q} \underbrace{-\left(\widehat{(}_{\mathrm{r}, \max }\right)^{2}}_{=: \iota_{\mathrm{r}}}
$$

and for the rotor voltage limit by

$$
0 \geq\left(\boldsymbol{i}_{\mathrm{r}}^{d q}\right)^{\top} \overline{\boldsymbol{V}} \boldsymbol{i}_{\mathrm{r}}^{d q}+2 \overline{\boldsymbol{v}}^{\top} \boldsymbol{i}_{\mathrm{r}}^{d q}+\bar{\nu}
$$

where the derivation of latter quadric is not stated due to space restrictions. Since $\frac{2}{3 \kappa}\left\|\boldsymbol{u}_{\mathrm{s}}^{d q}(t)\right\|=\widehat{u}_{\mathrm{s}, \max }$ for all $t \geq 0$, for a grid-connected stator of the DFIM, the stator voltage constraint in (4) boils down to the usage of $\widehat{u}_{\mathrm{s}, \max }$ in the stator voltage equation in (10). Moreover, the IM losses $p_{\mathrm{m}, \mathrm{L}}$ depend on the phase resistances which are assumed to be constant for simplicity. All machine parameters are listed in Tab. I.

Remark 1. Actually, resistances and therefore $p_{\mathrm{m}, \mathrm{L}}$ in (3) depend on stator \& rotor temperature, i.e. $\boldsymbol{R}_{\mathrm{s}}^{d q}\left(\vartheta_{\mathrm{s}}\right), \boldsymbol{R}_{\mathrm{r}}^{d q}\left(\vartheta_{\mathrm{r}}\right)$. In contrast to conventional LUT-based approaches, the analytical ORCC here can easily use these varying parameters online.

Table I: Parameters of the simulated but real DFIM.

\begin{tabular}{llr}
\hline Parameters & Symbol & Value \\
\hline Max. stator current & $\widehat{\imath}_{\mathrm{s}, \max }$ & $20.9 \mathrm{~A}$ \\
Max. rotor current & $\widehat{\imath}_{\mathrm{r}, \max }$ & $17 \mathrm{~A}$ \\
Max. stator voltage & $\widehat{u}_{\mathrm{s}, \max }$ & $\sqrt{2 / 3} \cdot 400 \mathrm{~V}$ \\
Max. rotor voltage & $\widehat{u}_{\mathrm{r}, \max }$ & $\sqrt{2 / 3} \cdot 400 \mathrm{~V}$ \\
Park angular velocity & $\omega_{\mathrm{p}}=-\omega_{\mathrm{s}}$ & $2 \pi 50 \frac{\mathrm{rad}}{\mathrm{s}}$ \\
Stator resistance & $R_{\mathrm{s}}$ & $0.72 \Omega$ \\
Rotor resistance & $R_{\mathrm{r}}$ & $0.55 \Omega$ \\
\hline
\end{tabular}

\section{B. Quadratic programming}

Applying the Lagrangian formalism to the QP

$$
\left.\begin{array}{rl}
\boldsymbol{i}_{\mathrm{r}, \mathrm{ref}}^{d q}:=\underset{\substack{\boldsymbol{i}_{\mathrm{r}}^{d q} \in \overline{\mathbb{S}} \\
\text { s.t. }}}{\left(\boldsymbol{i}_{\mathrm{r}}^{d q}\right)^{\top} \overline{\boldsymbol{T}} \boldsymbol{i}_{\mathrm{r}}^{d q}+2 \overline{\boldsymbol{t}}^{\top} \boldsymbol{i}_{\mathrm{r}}^{d q}+\bar{\tau}\left(m_{\mathrm{m}, \mathrm{ref}}\right)=0}
\end{array}\right\}
$$

where $\overline{\mathbb{S}}$ denotes the feasible set approximated at the current operating point, leads to three equations $\frac{\partial f}{\partial i_{\mathrm{r}}^{d}}=\frac{\partial f}{\partial i_{\mathrm{r}}^{q}}=\frac{\partial f}{\partial \lambda}=0$ with Lagrangian function $f\left(i_{\mathrm{r}}^{d q}, \lambda\right)$ and multiplier $\lambda$. Eliminating $\lambda$, an implicit solution (again a quadric, see [1]) is obtained for the Maximum Torque per (copper) Losses $\left(\mathrm{MTPL}_{\mathrm{Cu}}\right)$ strategy. Similarly, the Maximum Torque per (rotor) Current $\left(\mathrm{MTPC}_{\mathrm{r}}\right)$ strategy is obtained by replacing the overall copper loss quadric in (19) by the rotor copper loss quadric (13) only.

\begin{tabular}{|c|c|c|}
\hline Machine quadric & $\mathbb{Q}_{\mathrm{A}}$ or $\mathbb{Q}_{\mathrm{B}}$ & $\boldsymbol{A}, \boldsymbol{a}, \alpha$ \\
\hline Stator copper losses & $\overline{\overline{\mathbb{L}}_{\mathrm{s}, \mathrm{Cu}}}$ & $\overline{\boldsymbol{L}}_{\mathrm{s}, \mathrm{Cu}}, \overline{\boldsymbol{l}}_{\mathrm{s}, \mathrm{Cu}}, \rho_{\mathrm{s}, \mathrm{Cu}}$ \\
\hline Rotor copper losses & $\overline{\mathbb{L}}_{\mathrm{r}, \mathrm{Cu}}$ & $\overline{\boldsymbol{L}}_{\mathrm{r}, \mathrm{Cu}}, \mathbf{0}^{\top}, \rho_{\mathrm{r}, \mathrm{Cu}}$ \\
\hline Total copper losses & $\overline{\mathbb{L}}_{\mathrm{Cu}}$ & $\overline{\boldsymbol{L}}_{\mathrm{Cu}}, \overline{\boldsymbol{l}}_{\mathrm{Cu}}, \rho_{\mathrm{Cu}}$ \\
\hline Torque (equality) & $\overline{\mathbb{T}}\left(m_{\mathrm{m}, \mathrm{ref}}\right)$ & $\overline{\boldsymbol{T}}, \overline{\boldsymbol{t}}, \overline{\boldsymbol{\tau}}\left(m_{\mathrm{m}, \mathrm{ref}}\right)$ \\
\hline Stator current limit & $\partial \overline{\mathbb{I}}_{\mathrm{s}}\left(\widehat{\imath}_{\mathrm{s}, \max }\right)$ & $\overline{\overline{\boldsymbol{C}}_{\mathrm{s}}, \overline{\boldsymbol{c}}_{\mathrm{s}}, \bar{\iota}_{\mathrm{s}}\left(\widehat{\iota}_{\mathrm{s}, \max }\right)}$ \\
\hline Rotor current limit & $\partial \mathbb{I}_{\mathrm{r}}\left(\widehat{\imath}_{\mathrm{r}, \max }\right)$ & $\boldsymbol{C}_{\mathrm{r}}, \mathbf{0}^{\top}, \iota_{\mathrm{r}}\left(\widehat{\iota}_{\mathrm{r}, \max }\right)$ \\
\hline Rotor voltage limit & $\partial \overline{\mathbb{V}}\left(\widehat{u}_{\mathrm{r}, \max }\right)$ & $\overline{\boldsymbol{V}}, \overline{\boldsymbol{v}}, \bar{\nu}\left(\widehat{u}_{\mathrm{r}, \max }\right)$ \\
\hline MTPL $_{\mathrm{Cu}}$ strategy & $\overline{\mathrm{MTPL}}_{\mathrm{Cu}}$ & $\overline{\boldsymbol{M}}_{\mathrm{L}_{\mathrm{Cu}}}, \overline{\boldsymbol{m}}_{\mathrm{L}_{\mathrm{Cu}}}, \bar{\mu}_{\mathrm{L}_{\mathrm{Cu}}}$ \\
\hline $\mathrm{MTPC}_{\mathrm{r}}$ strategy & $\overline{\overline{M T P P C}}$ & $\overline{\boldsymbol{M}}_{\mathrm{C}_{\mathrm{r}}}, \overline{\boldsymbol{m}}_{\mathrm{C}_{\mathrm{r}}}, \bar{\mu}_{\mathrm{C}_{\mathrm{r}}}$ \\
\hline
\end{tabular}

All machine quadrics are summarized in Tab. II where $\partial$ denotes the boundary of an inequality constraint; e.g., the set $\partial \mathbb{I}_{\mathrm{r}} \subset \mathbb{I}_{\mathrm{r}}$ contains all $\boldsymbol{i}_{\mathrm{r}}^{d q}$ where the rotor current constraint is active, i. e. $\frac{2}{3 \kappa}\left\|\boldsymbol{i}_{\mathrm{r}}^{d q}\right\|=\widehat{\imath}_{\mathrm{r}, \max }$. Note that except $\partial \mathbb{I}_{\mathrm{r}}$, all sets depend on the actual operating point $\overline{\boldsymbol{i}}_{\mathrm{r}}^{d q}$ and thus, are indicated by symbols with overline.

Table II: Definition of the (approximated) machine quadrics.

The optimal solution is given by the intersection point of two quadrics, i. e. $i_{\text {ref }}^{d q}=\mathbb{Q}_{\mathrm{A}} \cap \mathbb{Q}_{\mathrm{B}}$, which can be calculated analytically (for details see [1]). The choices of $\mathbb{Q}_{\mathrm{A}}$ and $\mathbb{Q}_{\mathrm{B}}$ depend on which inequality constraints are active for the $\mathrm{QP}$ to be solved as shown in the next section.

\section{Operation Management}

Without any active inequality constraint or torque saturation, the optimal rotor current is equal to the intersection point $\overline{\mathbb{M T P L}}_{\mathrm{Cu}} \cap \overline{\mathbb{T}}\left(m_{\mathrm{m}, \mathrm{ref}}\right)$ [॰]. As illustrated in Fig. 3(a), the MTPL $_{\mathrm{Cu}}$ strategy [-] is the optimal compromise between stator copper losses [---] and rotor copper losses [---.] resulting in minimal machine copper losses [- - ]. The torque mainly depends on $i_{\mathrm{r}}^{d}$ such that the torque quadric $\overline{\mathbb{T}}$ moves to the left/right for in-/decreasing torque. In contrast to the $\mathrm{MTPL}_{\mathrm{Cu}}$ strategy, the $\mathrm{MTPC}_{\mathrm{r}}$ strategy [-] only minimizes the rotor copper losses. Hence, the intersection point $\mathbb{M T P C} \mathbb{C}_{r} \cap \mathbb{T}[\bullet]$ is only suboptimal.

For high torque reference magnitudes, the current constraints get active whereas the rotor voltage constraint can be neglected for this DFIM. Considering only the rotor current limit, the max./min. torque is given by the intersections of $\overline{\mathbb{M T P C}}_{\mathrm{r}}[-]$ with the rotor current limit set $\partial \mathbb{I}_{\mathrm{r}}[-]$ at $\overline{\mathbb{M T P C}}_{\mathrm{r}} \cap \partial \mathbb{I}_{\mathrm{r}}[\% / \mathrm{o}$. However, taking into account the stator current limit as well, the max./min feasible torque is given by 


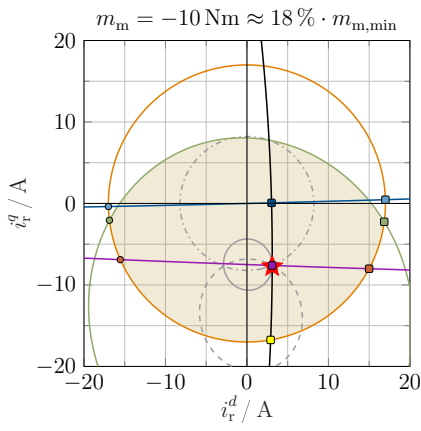

(a) $\mathrm{MTPL}_{\mathrm{Cu}}$

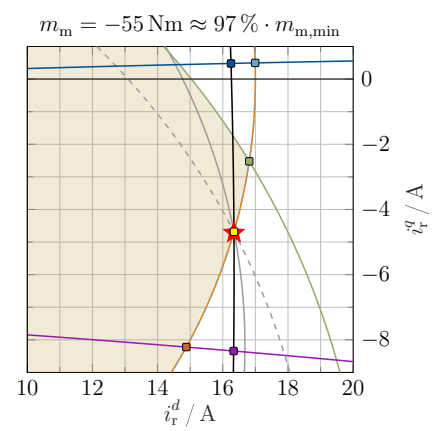

(b) $\mathrm{MC}_{\mathrm{r} \text { ext }}$
Figure 3: Current locus for different torques with quadrics for $\overline{\mathbb{L}}_{\mathrm{s}, \mathrm{Cu}}[---], \overline{\mathbb{L}}_{\mathrm{r}, \mathrm{Cu}}[\cdots \cdots], \overline{\mathbb{L}}_{\mathrm{Cu}}[-], \overline{\mathbb{T}}[-], \partial \overline{\mathbb{I}}_{\mathrm{s}}$ $[-], \partial \mathbb{I}_{\mathrm{r}}[-], \overline{\mathbb{M T P L}}_{\mathrm{Cu}}[-], \overline{\mathrm{MTPC}}_{\mathrm{r}}[-]$, feasible region $\overline{\mathbb{S}}:=\overline{\mathbb{I}}_{\mathrm{S}} \cap \mathbb{I}_{\mathrm{r}}[\quad]$ and reference currents $i_{\mathrm{r}, \text { ref }}^{d q}[\star]$.

the intersections of the stator current limit set $\partial \overline{\mathbb{I}}_{\mathrm{s}}[-]$ and $\partial \mathbb{I}_{\mathrm{r}}$ at $\partial \overline{\mathbb{I}}_{\mathrm{s}} \cap \partial \mathbb{I}_{\mathrm{r}}[\% / \mathrm{o}]$.

The max./min. feasible torque of the $\mathrm{MTPL}_{\mathrm{Cu}}$ strategy is reached at the intersection point $\mathbb{M T P L}_{\mathrm{Cu}} \cap \partial \mathbb{I}_{\mathrm{r}}[\% / 0]$. For higher torque magnitudes, the operating point $\overline{\mathrm{MTPL}}_{\mathrm{Cu}} \cap$ $\overline{\mathbb{T}}\left(m_{\mathrm{m}, \text { ref }}\right)$ [॰] of the $\mathrm{MTPL}_{\mathrm{Cu}}$ strategy is invalid and the optimal feasible operating point is given by the intersection $\partial \mathbb{I}_{\mathrm{r}} \cap \mathbb{T}[0]$ as visualized in Fig. $3(\mathrm{~b})$. During this so-called Maximum (rotor) Current $\left(\mathrm{MC}_{\mathrm{r}, \mathrm{ext}}\right)$ strategy, the rotor copper losses are constant due to constant rotor current amplitude. Only the stator current amplitude is increased to reach the max. feasible torque magnitude at $\partial \overline{\mathbb{I}}_{\mathrm{s}} \cap \partial \mathbb{I}_{\mathrm{r}}[\% / \bullet]$.

Using the gained knowledge about the DFIM characteristics and the minimization problem, an optimal operation management is designed as visualized by the decision tree in Fig. 4. The initial input parameters are the reference torque $\bar{m}_{\mathrm{m}, \mathrm{ref}}:=m_{\mathrm{m} \text {,ref }}$ and the (measured) rotor currents $\overline{\boldsymbol{i}}_{\mathrm{r}}^{d q}:=\boldsymbol{i}_{\mathrm{r}}^{d q}$. The magnetic flux linkages $\underline{\boldsymbol{\psi}}^{d q}\left(\overline{\boldsymbol{i}}_{\mathrm{r}}^{d q}\right)$, the steadystate differential inductances $\widetilde{\mathcal{L}}^{d q}\left(\overline{\boldsymbol{i}}_{\mathrm{r}}^{d q}\right)$ and, optionally, also other parameters such as the phase resistances $\overline{\boldsymbol{R}}^{d q}$ are updated based on the operating conditions and temperature variations.

In the next step, the torque is calculated at the intersection $\overline{\mathbb{M T P C}}_{\mathrm{r}} \cap \partial \mathbb{I}_{\mathrm{r}}$. If the solution is not feasible, i. e. $\boldsymbol{i}_{\mathrm{r}}^{d q^{*}} \notin \overline{\mathbb{S}}$, the torque is calculated again at $\partial \overline{\mathbb{I}}_{\mathrm{s}} \cap \partial \mathbb{I}_{\mathrm{r}}$ to obtain the new torque limits. If the reference torque exceeds these limits, the torque reference is saturated to $\bar{m}_{\mathrm{m}, \text { ref }}:=\operatorname{sat}_{\bar{m}_{\mathrm{m}, \text { min }}}^{\bar{m}_{\mathrm{max}}}\left(\bar{m}_{\mathrm{m}, \mathrm{ref}}\right)$ and the new operating point is set to the current solution at the active limit, i.e. $\overline{\boldsymbol{i}}_{\mathrm{r}}^{d q}:=\boldsymbol{i}_{\mathrm{r}}^{d q^{*}}$, before going back to the parameter update step. Otherwise, if the reference torque of the current iteration is within the limits, i. e $\bar{m}_{\mathrm{m}, \min } \leq \bar{m}_{\mathrm{m} \text {,ref }} \leq \bar{m}_{\mathrm{m}, \max }$, the final condition of the torque saturation $\bar{m}_{\mathrm{m}, \text { ref }}=\operatorname{sat}_{\bar{m}_{\mathrm{m}, \text { min }}}^{\frac{\bar{m}_{\mathrm{m}}}{m_{\mathrm{m}} \text {,ref }}}$ ) is checked. Latter condition ensures that the saturated torque reference has not fallen below the feasible limit at the final operating point. If this happens, the saturated torque reference for the next iteration is reset to the saturated initial torque reference, i. e. $\left.\bar{m}_{\mathrm{m}, \mathrm{ref}}:=\mathrm{sat}_{\bar{m}_{\mathrm{m}, \min }} \bar{m}_{\mathrm{m}, \mathrm{ref}}\right)$.

After completion of the torque saturation, the actual loss minimization starts with the calculation of $\overline{\mathbb{M T P L}}_{\mathrm{Cu}} \cap \overline{\mathbb{T}}$. If the solution $i_{\mathrm{r}}^{d q^{*}}$ is feasible, the $\mathrm{MTPL}_{\mathrm{Cu}}$ strategy is chosen, otherwise, overwriting $i_{\mathrm{r}}^{d q^{*}}$ with the solution of $\partial \mathbb{I}_{\mathrm{r}} \cap \overline{\mathbb{T}}$, leads to the $\mathrm{MC}_{\mathrm{r} \text { ext }}$ strategy. If the current operating point $\overline{\boldsymbol{i}}_{\mathrm{r}}^{d q}$ converged to the solution $\boldsymbol{i}_{\mathrm{r}}^{d q^{*}}$, i.e. $\left\|\boldsymbol{i}_{\mathrm{r}}^{d q^{*}}-\overline{\boldsymbol{i}}_{\mathrm{r}}^{d q}\right\| \leq \epsilon$ with some small threshold $\epsilon$, the optimal rotor current reference $i_{\mathrm{r}, \mathrm{ref}}^{d q}:=i_{\mathrm{r}}^{d q^{*}}$ is finally obtained [ $\left.\star \star\right]$.

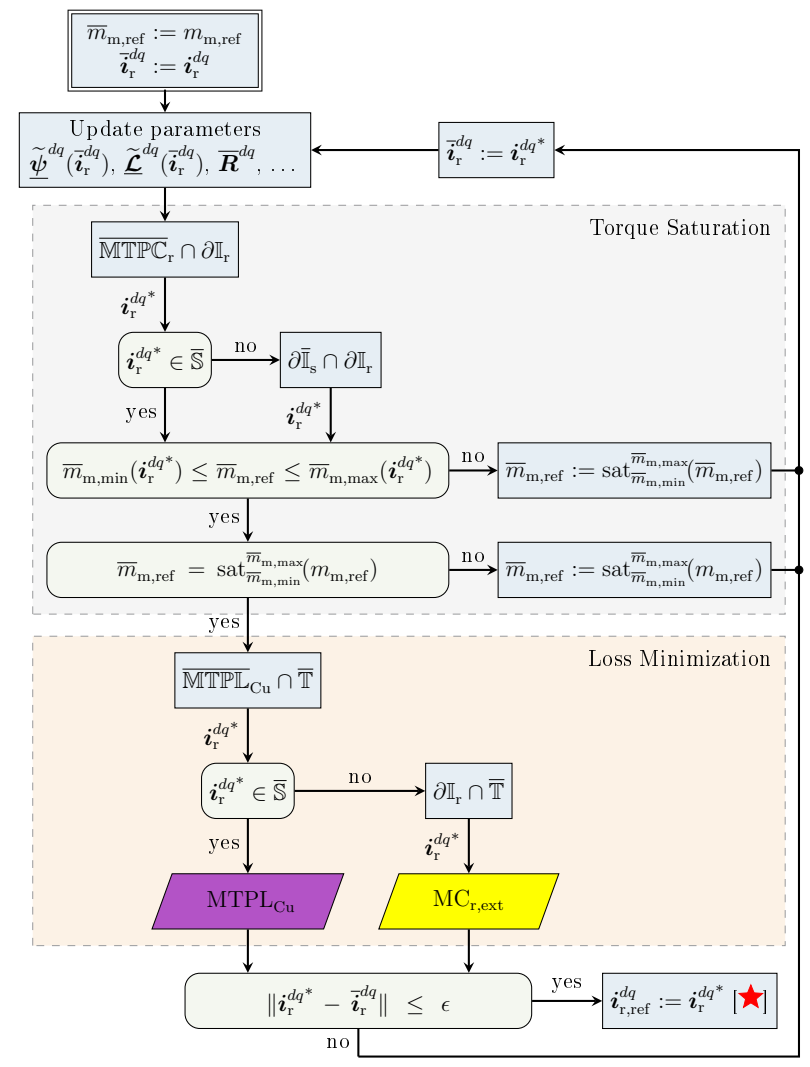

Figure 4: Decision tree for optimal operation management.

\section{Simulation RESUlts}

The nonlinear DFIM-model with flux linkage maps shown in Fig. 1, steady-state differential inductance maps shown in Fig. 2 and parameters listed in Tab. I as well as OFTC based on Tab. II and Fig. 4 are implemented and simulated in Matlab \& Simulink R2019b. The reference torque is provided by an outer-loop speed controller and the OFTC computes the reference currents for the inner-loop rotor current controller. For the simulated scenario, the DFIM is operated in generator mode and the slip is varied from $30 \%$ super-synchronous to $0 \%$.

Fig. 5 shows the simulated time series with gradually increasing load torque from zero to its final value $m_{1}=$ $30 \mathrm{Nm} \approx 53 \% \cdot\left|m_{\mathrm{m}, \min }\right|$ at $t=0.2 \mathrm{~s}$. At the beginning, the speed reference is constant but is gradually decreased from $\omega_{\mathrm{m}, \mathrm{ref}}=1.3 \pi 50 \frac{\mathrm{rad}}{\mathrm{s}}$ at $t=0.3 \mathrm{~s}$ to its final value $\omega_{\mathrm{m}, \mathrm{ref}}=\pi 50 \frac{\mathrm{rad}}{\mathrm{s}}$ at $t=0.45 \mathrm{~s}$. During the time interval $305 \mathrm{~ms} \leq t \leq 467 \mathrm{~ms}$ the OFTC choses $\mathrm{MC}_{\mathrm{r} \text {,ext }}$ strategy and, at the remaining time, the $\mathrm{MTPL}_{\mathrm{Cu}}$ strategy. With increasing torque magnitude, the $d$-component of the rotor 

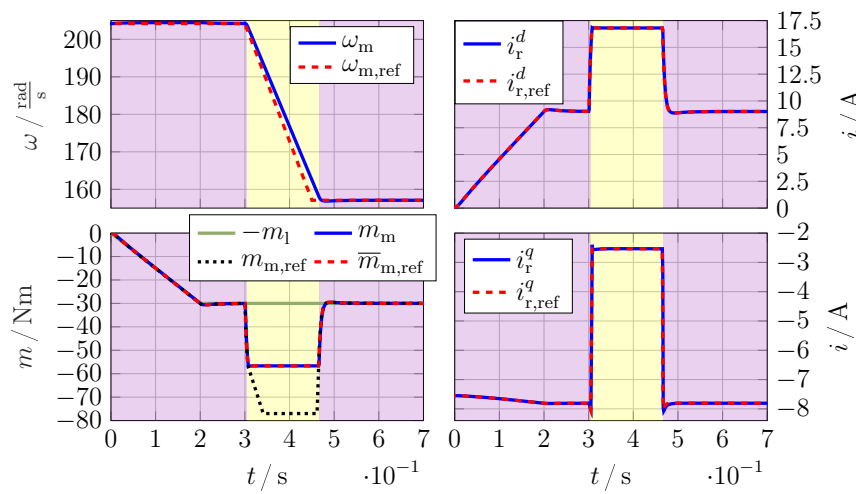

Figure 5: Simulation results with transitions between the operation strategies $\mathrm{MTPL}_{\mathrm{Cu}}\left[\mathrm{]}\right.$ and $\mathrm{MC}_{\mathrm{r} \text {,ext }}[$ ].

current reference $i_{\mathrm{r}, \text { ref }}^{d}$ increases for both strategies. In contrast, the $q$-component decreases from $i_{\mathrm{r}, \mathrm{ref}}^{q} \approx-7.55 \mathrm{~A}$ at $t=0 \mathrm{~s}$ to $i_{\mathrm{r} \text {,ref }}^{q}=-8.04 \mathrm{~A}$ at $t=305 \mathrm{~ms}$ during the $\mathrm{MTPL}_{\mathrm{Cu}}$ strategy. However, $i_{\mathrm{r}}^{q}$ rapidly increases $-\mathrm{i}$. e. the magnitude of $i_{\mathrm{r}}^{q}$ rapidly decreases - after switching to the $\mathrm{MC}_{\mathrm{r} \text {,ext }}$ strategy in order to reach the max. torque magnitude with the max. feasible $i_{\mathrm{r}}^{d}$. During the deceleration phase, the reference torque $m_{\mathrm{m} \text {,ref }}$ is saturated to $\bar{m}_{\mathrm{m} \text {,ref }}$ resulting in a notable deviation between actual and reference speed.

In Fig. 6 , the efficiency of the OFTC $\eta_{\mathrm{OFTC}}:=\frac{p_{\mathrm{s}}+p_{\mathrm{r}}}{p_{\mathrm{m}}}$ is compared with the efficiency of conventional torque control with $i_{\mathrm{s}}^{q}=0$ or $i_{\mathrm{r}}^{q}=00^{2}$ For $i_{\mathrm{s}}^{q}=0$, a large negative reactive rotor current $i_{\mathrm{r}}^{q}<-13.8 \mathrm{~A}$ is required such that the torque is limited to $m_{\mathrm{m} \text {,min }} \approx-29 \mathrm{Nm}$ due to rotor current limitation. For $i_{\mathrm{r}}^{q}=0$, the torque is limited to $m_{\mathrm{m} \text {,min }} \approx-50.5 \mathrm{Nm}$ at $i_{\mathrm{r}}^{d} \approx 15 \mathrm{~A}$ due to stator current limitation, cf. Fig. 3 (b). At approximately the same torque, the OFTC switches from MTPL $L_{\mathrm{Cu}}$ to $\mathrm{MC}_{\mathrm{r}, \text { ext }}$. With the $\mathrm{MC}_{\mathrm{r} \text {,ext }}$ strategy, the OFTC reaches the max. feasible torque magnitude of $m_{\mathrm{m}, \min } \approx-56.7 \mathrm{Nm}$. Note the slightly increased curvature at the end of the $\eta_{\text {OFTC }}$-line near $m_{\mathrm{m}, \mathrm{min}}$ in the left plot of Fig. 6 as the $M_{r, \text { ext }}$ strategy diverges from the $M T P L_{C u}$ strategy.

The right plot shows the difference $\Delta \eta$ between the efficiency of OFTC and torque control with $i_{\mathrm{s}}^{q}=0$ or $i_{\mathrm{r}}^{q}=0$. The efficiency using OFTC is increased by more than $1 \%$ in the whole feasible generator torque range. For torques $m_{\mathrm{m}} \geq-10 \mathrm{Nm} \approx 18 \% \cdot m_{\mathrm{m}, \min }$, the efficiency is increased by more than $\Delta \eta>3 \%$.

\section{CONClusion}

A generic and analytical solution for the optimal feedforward torque control problem (OFTC) of nonlinear induction machines (IMs) was presented considering stator and rotor copper losses. An operation management was designed for a doubly-fed induction machine (DFIM) which saturates the

\footnotetext{
${ }^{2}$ Actually, the reactive power reference at the point of common coupling (PCC) of WECS depends on the grid demand. However, unitary power factor operation with $i_{\mathrm{s}}^{q}=0$ is commonly considered, but using the grid-side converter (GSC) for reactive power control is also an option [15]. For OFTC, it is assumed that the GSC compensates the reactive power of the DFIG at the PCC (if required).
}

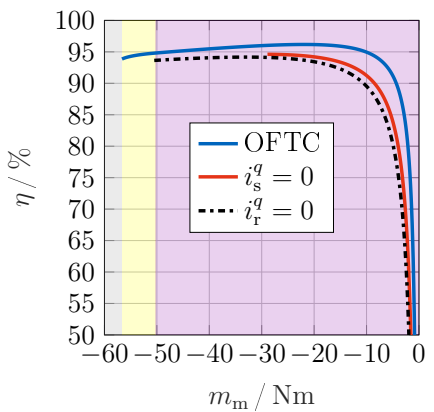

(a)

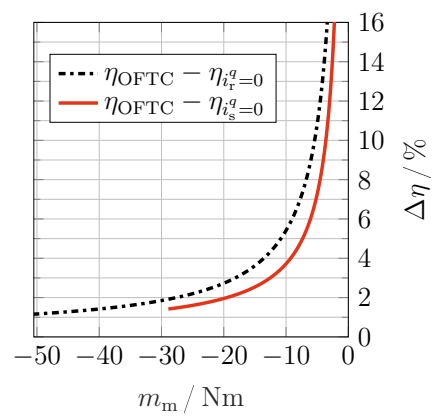

(b)
Figure 6: At $30 \%$ slip (super-synchronous): (a) Efficiency of the OFTC $\eta_{\mathrm{OFTC}}:=\frac{p_{\mathrm{s}}+p_{\mathrm{r}}}{p_{\mathrm{m}}}$ with infeasible area [ ], $\mathrm{MTPL}_{\mathrm{Cu}}\left[\mathrm{]}\right.$ and $\mathrm{MC}_{\mathrm{r}, \text { ext }}\left[p_{\mathrm{m}}\right]$ strategy areas as well as efficiency of conventional torque control with $i_{\mathrm{s}}^{q}=0$ or $i_{\mathrm{r}}^{q}=0$, and (b) efficiency enhancement $\Delta \eta:=\eta_{\text {OFTC }}-\eta_{i_{\mathrm{s}}^{q}=0 / i_{\mathrm{r}}^{q}=0}$.

reference torque and choses the optimal operating strategy. The OFTC accurately tracks the reference torque and minimizes all copper losses, resulting in significantly increased efficiency compared with conventional unitary power factor control. Moreover, the max. feasible torque is reached taking into account rotor and stator current \& voltage limits, respectively. Future research will include further types of losses, such as iron and converter losses, and validate the theoretical and simulative results in the laboratory.

\section{REFERENCES}

[1] H. Eldeeb, C. M. Hackl, L. Horlbeck, and J. Kullick, "A unified theory for optimal feedforward torque control of anisotropic synchronous machines," International Journal of Control, 2017.

[2] J. Machowski, Z. Lubosny, J. W. Bialek, and J. R. Bumby, Power system dynamics. stability and control. John Wiley \& Sons, 2020.

[3] M. Liserre, R. Cardenas, M. Molinas, and J. Rodriguez, "Overview of multi-MW wind turbines and wind parks," IEEE, vol. 58, pp. 1081-1095, Apr. 2011.

[4] A. G. Abo-Khalil, H.-G. Park, D.-C. Lee, S.-P. Ryu, and S.-H. Lee, "Loss minimization control for doubly-fed induction generators in variable speed wind turbines," IEEE, Nov. 2007.

[5] B. Zhang, W. Hu, and Z. Chen, "Loss minimizing operation of doubly fed induction generator based wind generation systems considering reactive power provision," IEEE, Oct. 2014.

[6] M. N. Soares, J. Gyselinck, L. G. B. Rolim, J. Helsen, and Y. Mollet, "Loss minimisation strategy for DFIG in wind turbine considering iron losses," in 2018 IEEE International Conference on Industrial Technology (ICIT), IEEE, feb 2018.

[7] S. Puchalapalli and B. Singh, "Optimal RSC control for loss reduction in wind turbine driven DFIG-grid system," IEEE, Oct. 2020.

[8] N. Karakasis, E. Tsioumas, N. Jabbour, A. M. Bazzi, and C. Mademlis, "Optimal efficiency control in a wind system with doubly fed induction generator," IEEE Transactions on Power Electronics, vol. 34, pp. 356-368, jan 2019.

[9] S. A. Odhano, R. Bojoi, A. Boglietti, Ş. G. Roşu, and G. Griva, "Maximum efficiency per torque direct flux vector control of induction motor drives," IEEE Transactions on Industry Applications, vol. 51, pp. 4415-4424, Nov 2015.

[10] J. Kullick and C. M. Hackl, "Generic machine identification and maximum efficiency operation of induction machines," arxiv, 2019.

[11] C. M. Hackl, J. Kullick, and N. Monzen, "Generic loss minimization for nonlinear synchronous machines by analytical computation of optimal reference currents considering copper and iron losses," in Special Session - 22nd IEEE International Conference on Industrial Technology (ICIT), IEEE, Jan. 2021.

[12] C. Bals and D. Gerling, "Reduced order model of the induction machine derived from finite-element-analysis," in 2016 XXII International Conference on Electrical Machines (ICEM), pp. 2038-2044, 2016.

[13] J. Nocedal and S. Wright, Numerical Optimization. Springer Science \& Business Media, 2006.

[14] C. Hackl, J. Kullick, and N. Monzen, "Optimale Betriebsführung für nichtlineare Synchronmaschinen," in Elektrische Antriebe - Regelung von Antriebssystemen (J. Böcker and G. Griepentrog, eds.), Springer-Verlag, 2020

[15] M. Debouza and A. Al-Durra, "Grid ancillary services from doubly fed induction generator-based wind energy conversion system: A review," IEEE Access, vol. 7, pp. 7067-7081, 2019. 